International Journal of Performability Engineering, Vol. 13, No. 1, January 2017, pp. 03-18

(C) Totem Publisher, Inc., 4625 Stargazer Dr., Plano,

Printed in U.S.A.

\title{
Numerical Investigation of the Inner Flow in Centralized Fluffs Collection using CFD
}

\author{
C.N. JAYAPRAGASAN*, and K. JANARDHAN REDDY \\ School of Mechanical and Building Sciences, VIT University, Chennai, INDIA
}

(Received on September 03, 2016, revised on October 16, 2016)

\begin{abstract}
Centralized collection system is strongly influenced by the flow of fluffs inside the pipeline and geometry parameters. Therefore, it has become necessary to find out the numerical investigation of flow inside the pipe line. In this study, the performance effect of fluid flow inside the centralized waste collection pipeline was numerically investigated with different configuration of inlet points which were opened at the same time of discharge. Centralized blower collects the fluffs from the collection box inlets which were delivered by an Industrial cleaner. Numerical investigations on five cases are done with the help of FLUENT software of computational fluid dynamics. CFD was used to determine the significance of output parameters with respect to the flow inside the pipe. This study helps to understand the flow behavior inside the pipeline and to compare the output parameters with experimental investigation. These investigation results are used to develop alternative solution to reduce the energy consumption of centralized collection system. Results indicates that the output parameters of inlet collection points 1,4,8 and 12 which were discharged at the same time gives the maximum power consumption. Thus it has been chosen for the experimental investigation of centralized waste collection system in order to get an optimized alternative solution for better energy saving.
\end{abstract}

Keywords: Centrifugal blower, Computational fluid dynamics (CFD) and Centralized pipeline

\section{Introduction}

Centralized waste collection systems are widely used in industries for collection of fluffs from the number of collection boxes. It is an important aspect for industries to improve the fluffs collection efficiency and consequently the running cost. The industrial cleaner sucks the cotton fluffs from the floor and discharged into the collection box at one end of the railing. The collected fluffs are sucked by an automatic centralized waste collection system. The centralized waste collection system is recommended for the use where there are at least 4 or 5 industrial cleaners running in the plant.

The capacity of the centralized blower motor is directly proportional to the number of industrial cleaners running on the track, size of the connecting pipes, the distance between the machines and the location of the blower unit. This investigation is an analytical methodology considering the number of collection boxes opened at various stages to examine the flow field performance of the centralized waste collection system.

To validate the data, the test cases were modelled and investigated. Hence a comprehensive numerical study is carried out to optimize the performance of centralized collection system and energy consumption.

This study focuses on numerical flow analysis to measure the output parameters on five cases of inlet points in centralized collection system.

*Corresponding author's email: cnjp68@gmail.com 


\author{
Notation \\ CFD computational fluid dynamics \\ D outer diameter of pipe, $\mathrm{mm}$ \\ $\mathrm{P}_{\mathrm{tf}} \quad$ total pressure developed by blower in Pascal, $\mathrm{Pa}$ \\ Q discharge, cfm \\ cfm cubic feet per meter \\ $\mathrm{mm}$ in wc millimeters of water column
}

\title{
2. Literature survey and related work
}

The performance of the fan is governed by different fan laws. Hence lot of emphasis is given to understand the basic theory of fans, their types and their working. The selection of critical parameters is very essential while determining the performance of the fans [1]. When carrying out the design optimization of centrifugal fans, a complete design methodology is to be followed. It is also necessary to develop a numerical procedure whose results can be compared with the results from CFD simulation [2].

Parametric studies are carried out to compute the power coefficient along with flow coefficient and efficiency. Finally a systematic and reliable strategy is required to correctly interpret the CFD solutions and compare them with the experimental results [3].

While performing the experimental and numerical study the essential parameters to be determined are detailed flow visualization, torque calculation, efficiency estimation and noise analysis. The results are fairly accurate using to solve 3D Navier-strokes equation and adopting the second-order upwind scheme for $\mathrm{k}-\varepsilon$ calculations [4].

Simulation, or more specifically numerical simulation is a powerful tool for modeling of engineering, social and other system where the traditional analytical of graphical technique became very complex [5]. Simulation is usually prepared approach to model and predict the life cycle characteristics such as cost and availability of large populations based on design and support parameters [6].

Experimental and numerical analysis of multiphase flow in pipeline and blower have been shown in existing literature. The effect of volume fraction, expansion and contraction of pipeline has been studied through experimental as well as numerical methods. As well as the effect of blade diameter and blade width on mass flow rate has been studied.

The multi-phase flow of gas and solid in curved $90^{\circ}$ horizontal square bend pipe is investigated. Sand is used as the solid phase in the particle diameter of $63 \mu \mathrm{m}, 125 \mu \mathrm{m}, 200$ $\mu \mathrm{m}, 400 \mu \mathrm{m}, 630 \mu \mathrm{m}, 800 \mu \mathrm{m}$ and $1250 \mu \mathrm{m}$. Numerical analysis is performed by Lagrangianparticle tracking model for predicting particle trajectories of dispersed phase, and $\mathrm{k}-\varepsilon$ model for predicting the turbulent (air-sand) flows in bends. In this analysis one way coupled Lagrangian solid particle tracking is applied to find and evaluate particle dynamics within elbow and straight ducts because solid motion is negligible influence in fluid flow. Based on the analysis 20000 particles is tracked based on giving particle size distribution. The solid loading ratio of 1.2 is used in this experiment. Theoretical and experimental data are compared for the particle trajectories and velocity vectors. The results concluded that the concentration of particles decreases near the inner wall and increases at the outer wall with the increase of particle diameter [7], from the above paper it was seen that the $\mathrm{k}-\varepsilon$ model was the most appropriate model to recreate the turbulence in the pipe. So $\mathrm{k}-\varepsilon$ model was used in the whole project to model the turbulence in the flow. 
CFD simulation is performed to investigate core annular flow through sudden expansion and contraction of pipes. Volume of fluid technique is used in CFD to solve core annular flow of lubricating water and oil. The transient simulation with the time step of $0.01 \mathrm{~s}$ was carried out. In this study, Continuity equation has been discretized by PRESTO while momentum, turbulent kinetic energy and dissipation rate equations are discretized by the first order upwind method. PISO algorithm is used for pressure, velocity coupling. Then the experimental result is compared with the simulation result. The study has been performed to produce the profile of pressure, velocity and volume fraction. In the result shows that the volume fraction of oil seems to be constant after 10 $\mathrm{L} / \mathrm{D}$ in case of contraction while in case of expansion it increases from up to downstream [8].The gas and solid particle flow in 2-dimensional vertical channel $(0.1 \mathrm{x} 1 \mathrm{~m})$ is investigated. The mass flow rate of fluid phase is fixed and volume fraction was considered between 0.01 to 0.0001 with the particle diameter of $252 \mu \mathrm{m}$. From the results of two fluids and MFIX - QMOM method [9], it was seen that transient simulation with a time step of 0.01 gives optimum results for the flow and the volume of fluid. This model was the best multiphase model for study of flow in pipe.

Radial blower is good for dust extraction process. For that rotational frequency plays main role to improve the mass flow rate. The rotational frequency considered are 3550,2950 and 2350RPM. While increasing rotational frequency of the fan, mass flow rate and efficiency of blower is also increased [10]. Comparison made from three different types of impeller namely forward, backward and radial based on three types of parameters are pressure, velocity and noise of the centrifugal blower by numerical analysis. From this analysis, it is concluded that backward impeller generates less noise and high pressure compared to other forward and radial impellers [11]. From the above paper, radial blower is found to be more efficient than forward and backward blower. So the radial blower was chosen for the study.

For optimizing the backward- swept blade centrifugal fan, 12 numbers of blades are selected. Three types of parameter are chosen for improving performance is inlet blade angle, outlet blade angle and number of blades is selected. With the help of analyzing the results, optimization done based on the Taguchi method [12].For the study of the effect of impeller width on mass flow rate of centrifugal blower, three different CAD models were made with an impeller width of 17,20,25 mm. It is observed that when impeller width is increased from $17 \mathrm{~mm}$ to $20 \mathrm{~mm}$ mass flow rate also increased from 0.118 to 0.134 $\mathrm{Kg} / \mathrm{Sec}$. Also, when impeller width is further increased by $1.5 \mathrm{~mm}$, the mass flow rate decreased to $0.126 \mathrm{Kg} / \mathrm{Sec}$ [13]. From the above paper, it shows that impeller width with number of blades will plays main role for change in mass flow rate.

While considering practical problem, the best way to find correct numerical result is turbulence model. Momentum equation and continuity equation are considered for the good numerical result [14]. Three types of axial gap between the inlet nozzle and impeller (two smaller than the impeller diameter and one bigger than impeller diameter) are used. From this analysis, it concluded that the blower with axial gap and inlet diameter is reduced, then efficiency is increased [15]. From the above paper, the axial gap also plays main role in improving efficiency of blower.

In a few research paper of multi flow analysis, it was observed that the fluid and solid particles had been considered whereas the mixed flow of fluid and cotton particles had not been taken into consideration for analysis. Even though little scientific documentation related to mixed flow was observed, no one has attempted to formulate a numerical and an 
experimental investigation of fluid and cotton particles to find the flow field performance in the centralized pipe line system. The aim of the present paper has focused to investigate the output parameters of flow field inside the pipe passage through the numerical simulation.

\section{Research Methods}

The Research methods comprises of following steps:

- Literature Survey

- Modeling approach

- Modeling, Meshing of centralized collection system

- Numerical Simulation

\subsection{Modeling approaches}

Volume of fluid (VOF) method approach belongs to the Euler-Euler framework where all phases are treated as continuous, but in contrary to the previous presented models the VOF model does not allow the phases to be interpenetrating. The VOF method uses a phase indicator function, sometimes also called a colour function, to track the interface between two or more phases. The indicator function has value one or zero when a control volume is entirely filled with one of the phases and a value between one and zero if an interface is present in the control volume. Hence, the phase indicator function has the properties of volume fraction. The transport equations are solved for mixture properties without slip velocity, meaning that all field variables are assumed to be shared between the phases. To track the interface, an advection equation for the indicator function is solved. In order to obtain a sharp interface the discretization of the indicator function equation is crucial.

As the focus of the VOF method is to track the interface between two or more phases it is suitable for flows with sharp interfaces, such as slug, stratified or free-surface flows. The equations solved in the VOF method are shown in Eq. (1) and (2).

$$
\begin{aligned}
& \frac{\partial \rho_{m} u}{\partial_{t}}+\nabla\left(\rho_{m} u\right)=0 \\
& \frac{\partial \rho_{m} u}{\partial_{t}}+\nabla\left(\rho_{m} u u\right)=\nabla P+\nabla \tau+\rho_{m} g+S=0
\end{aligned}
$$

The simulations were based on an actual working condition of centralized collection system containing air-cotton mixtures in a horizontal pipe line with branches. The subscript $\mathrm{m}$ refers to mixture properties.

\subsection{Modeling and Meshing}

The Fluent solver is based on the center node FVM discretization technique and offers both segregated and coupled solution methods. There are three Euler-Euler multiphase models are available; the Eulerian model, the mixture model and the VOF model. The discretization of 
the volume fraction equation is crucial in a VOF method to keep the interface sharp. The choice of discretization method can have a great influence on the results in other multiphase models as well.

To resolve this issue, Fluent has a number of discretization techniques implemented specifically for the volume fraction equation. In this analysis, the VOF approach has been used to find the flow field inside the pipeline of the centralized collection system. The domain and the meshes were created using ANSYS ICEM CFD.

In order to maintain the effective velocity for the entire length of the main pipeline, the diameter of the pipe has been reduced gradually to the certain length such as $140 \mathrm{~mm}, 160$ $\mathrm{mm}$ and $200 \mathrm{~mm}$. All " $\mathrm{y}$ " joints of $\varnothing 110 \mathrm{~mm}$ with $45^{\circ}$ angle are connected to the collection boxes and main pipe. The one end of the main pipe $(\varnothing 200 \mathrm{~mm})$ has been connected to the inlet mouth of 5HP blower and other end (Ø $140 \mathrm{~mm}$ ) has been closed by $\varnothing 140 \mathrm{~mm}$ end cover. The distance between the collection boxes ("y" joints) are maintained at 2 meters and twelve such boxes are connected with the main pipe through "y" joint. The cotton flags are transferred by air from the collection box to the blower through the main pipe. The centralized pipeline has 12 inlets and one outlet which is connected to 5 HP blower and it sucks the fluffs along with air from all the inlets. A block-structured meshing approach was used to create meshes with only hexahedral /quad cells in ICEM CFD with the mesh count of 5.5 million.

Several combinations of cases were simulated and parameters were systematically changed in order to investigate their effect on the result. In that, the effect of turbulence model, drag law, discretizing scheme for the gas volume fraction (GVF) equation and phase formulation was investigated. Analysis of the pipeline was carried out in FLUENT software. The schematic arrangement of the centralized pipe line connection is given in Figure 1.

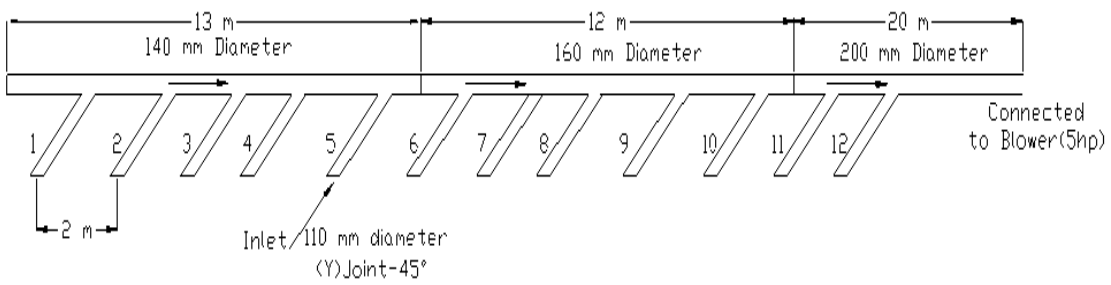

Fig. 1: Schematic arrangement of centralized collection pipeline

\subsubsection{Boundary Conditions}

The numerical investigation of centralized collection system with 12 inlet branches flow fields has been carried out. The points which have been considered for the numerical studies are in multiphase, such as air and cotton are selected as primary and secondary phase respectively. The density and viscosity of air were $1.225 \mathrm{Kg} / \mathrm{m}^{3}$ and $1.789 \mathrm{e}-05$ $\mathrm{Kg} / \mathrm{m}^{3}$ respectively. The surface tension of the air-cotton was $0.072 \mathrm{~nm}-1$. Turbulence model was chosen as K-epsilon Realizable model with scaled wall function. The inlet is considered as inlet-vent and outlet is considered as pressure outlet. 
The hydraulic diameter of the inlet was $0.11 \mathrm{~m}$ and the outlet main pipe was $0.14 \mathrm{~m}$, $0.16 \mathrm{~m}$ and $0.2 \mathrm{~m}$. As per the experimental results [15] from the previous study, the discharge of cotton fluffs by the cleaner for one cycle was 9.7 gms in one collection box. The blower experimental value of suction pressure was $2070 \mathrm{~Pa}$. Studied the velocity and pressure flow fields of centralized collection system with the following cases at a single time discharge,

- Twelve inlet points were opened.(1,2,3 to 12$)$

- First inlet point opened. (1)

- First and Fourth inlet points were opened. (1 and 4)

- First, Fourth, and Eighth inlet points were opened $(1,4$ and 8$)$

- First, Fourth, Eighth and twelfth inlet points (1,4,8 and 12) were opened at a single time discharge.

\section{Numerical analysis of centralized collection system}

\subsection{Twelve collection box inlet points were opened at a single time discharge}

Numerical simulations of the three-dimensional turbulent flow field was carried out in a horizontal centralized collection pipeline system with considering twelve collection box inlets were opened at a single time discharge. The computational domain and meshed model of the pipe are shown in Figure 2 and 3.

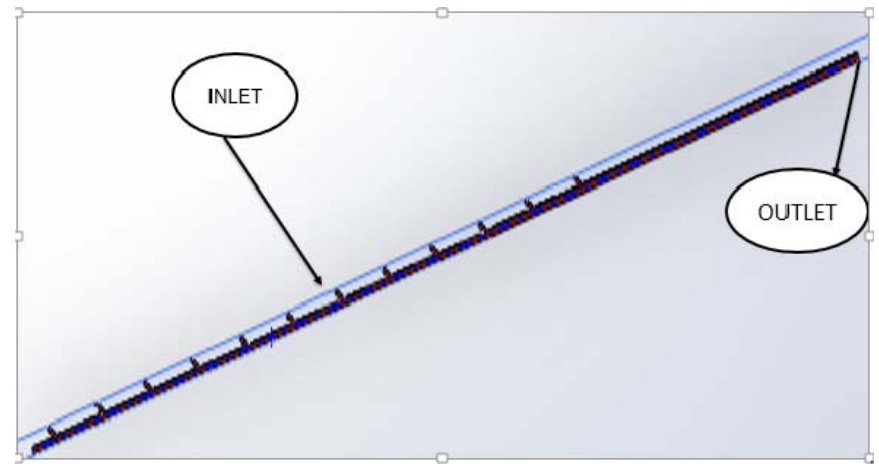

Fig. 2: Original computational domain

A block-structured meshing approach was used to create meshes with only hexahedral /quad cells in ICEM CFD with the mesh count of 5.5 million. 


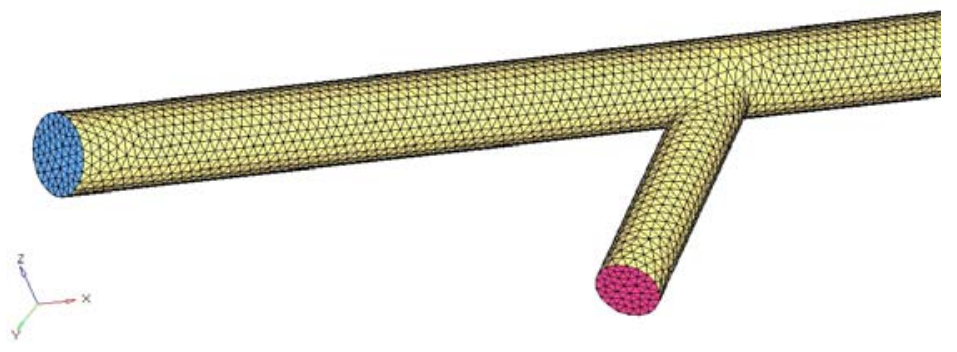

Fig. 3: Meshed 3D model of pipe

The volume fraction profile of air shows a peak in the front inlet connection of the centralized pipeline which was nearest to the blower. It was observed that the cotton volume fraction is 0.00053 at inlet 1 and 2 . Further, it was gradually reduced up to twelfth inlet connection and it was observed at 0.000025 . The collection of cotton fluffs is only $9.7 \mathrm{gms}$ in one cycle of operation [15], so the volume fraction profile for cotton is very less when compared to the air.

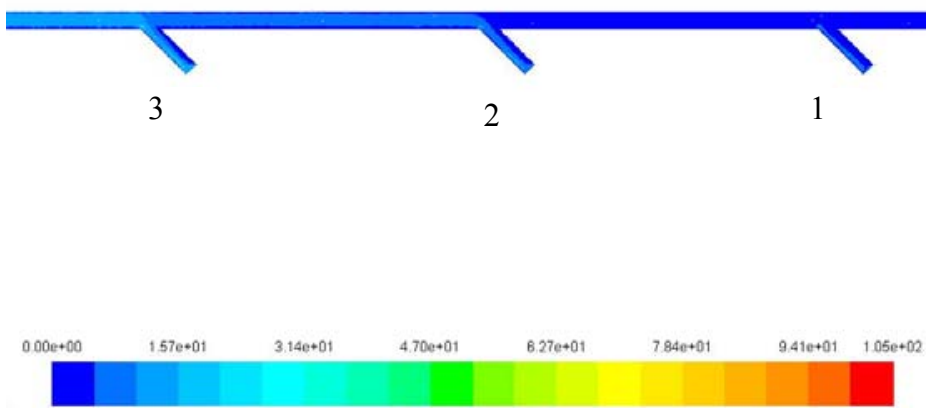

Fig. 4: Velocity contours for inlet points 1,2 and 3

Figures 4 and 5 show the velocity contour of inlets $1,2,3,10,11$ and 12 . At the inlets 1,2 and 3 , the velocity of the mixture was observed as $5.66 \mathrm{~m} / \mathrm{s}, 8.95 \mathrm{~m} / \mathrm{s}$ and $13.4 \mathrm{~m} / \mathrm{s}$ respectively. Similarly the velocity of the mixture at inlets 4,5 , and 6 was observed as 14.92 $\mathrm{m} / \mathrm{sec}, 16.41 \mathrm{~m} / \mathrm{sec}$ and $26.85 \mathrm{~m} / \mathrm{sec}$ respectively. It was clearly shown that the velocity was low at inlet 1 and gradually increases in other inlets. 


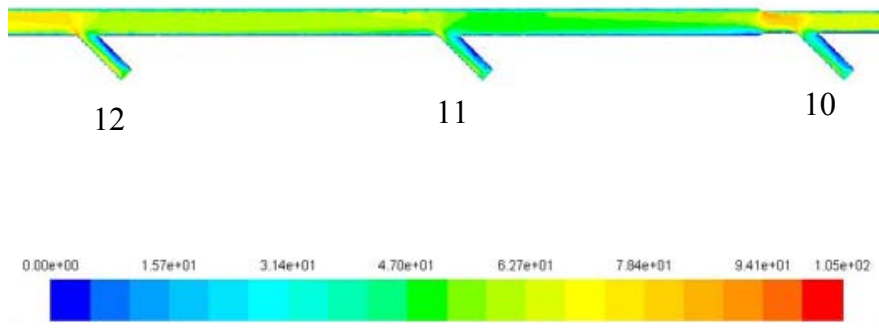

Fig. 5: Velocity contours for inlets 10,11 and 12

Similarly the velocity contour at the inlets 7,8 and 9 was observed as $32.9 \mathrm{~m} / \mathrm{s}, 29.8$ $\mathrm{m} / \mathrm{s}$ and $41.77 \mathrm{~m} / \mathrm{sec}$ respectively. The velocity of the inlets 10,11 , and 12 was observed as $43.7 \mathrm{~m} / \mathrm{sec}, 44.76 \mathrm{~m} / \mathrm{sec}$ and $55.21 \mathrm{~m} / \mathrm{sec}$ respectively. It was clearly shown that the velocity was gradually increased from inlet 1 to the inlet 12 in the range from $5.66 \mathrm{~m} / \mathrm{sec}$ to $55.21 \mathrm{~m} / \mathrm{sec}$ and same has been maintained in the main pipe.

\subsection{First collection box inlet was opened at a single time discharge}

Numerical simulations were carried out for a horizontal centralized collection pipeline system by considering first collection box inlet only open and remaining are closed while the blower is running.

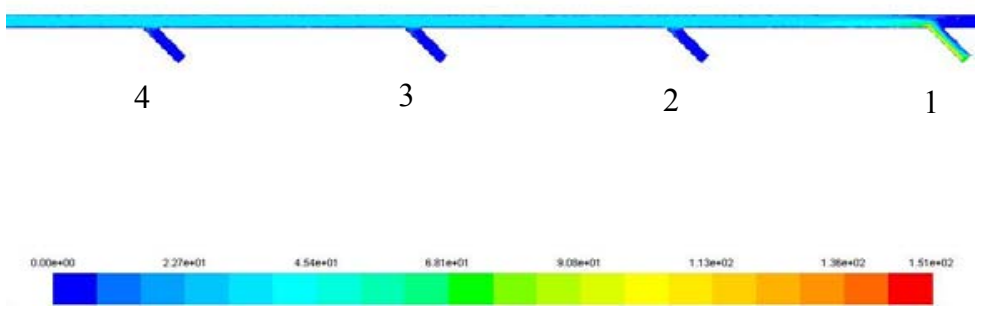

Fig. 6: Velocity contours for inlets 1-4 


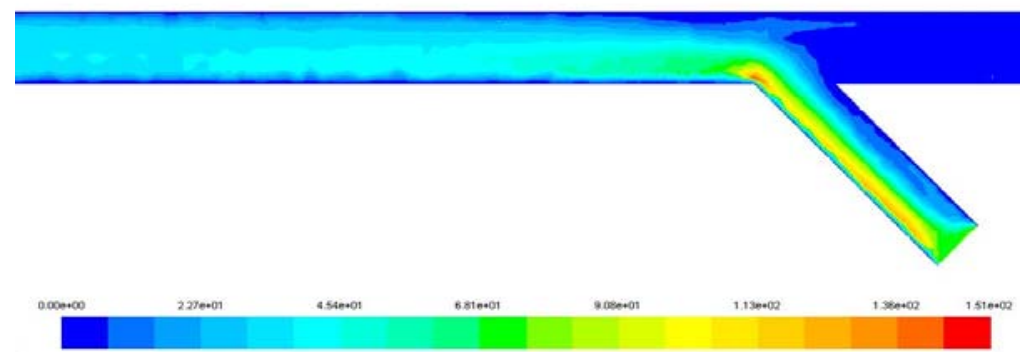

Fig. 7: Velocity contours for inlet 1

Figures 6 and 7 show the contour of static pressure and velocity at inlet 1 and it was observed as $948.7 \mathrm{~Pa}$ and $76.39 \mathrm{~m} / \mathrm{sec}$ respectively. It was clearly shown that the velocity was high at inlet 1 and gradually decreases in the main pipe in the range of $76.39 \mathrm{~m} / \mathrm{sec}$ to 35.7 $\mathrm{m} / \mathrm{sec}$ in between inlet 1 to 2 .

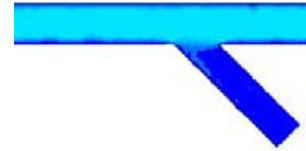

2

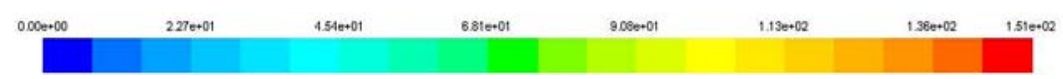

Fig. 8: Velocity contours for inlets 1 and 2 


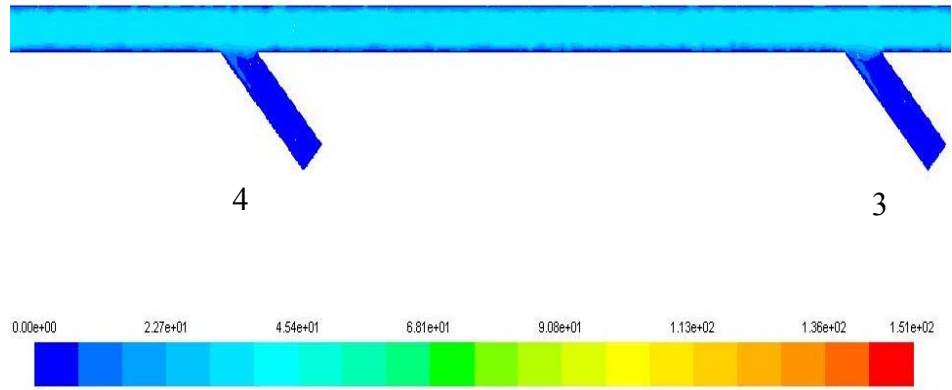

Fig. 9: Velocity contours for inlets 3 and 4

Figures 8 and 9 show the velocity contour of inlets 1 to 2 and inlets 3 to 4 respectively. It was observed that the velocity between the inlets 1 to 4 varies in the range from $35.7 \mathrm{~m} / \mathrm{sec}$ to $36.4 \mathrm{~m} / \mathrm{sec}$ which shows a minor difference. The inlets 2,3 and 4 was in closed condition and observed that the velocities of the points were minimum. Similarly the numerical analysis of other cases (1-4 open, 1-4-8 and 1-4-8-12 points are opened at single time discharge) were carried out and results were tabulated.

\subsection{Inlets first, fourth, eighth and twelfth are opened at a single time discharge:}

The detailed numerical analysis of air and cotton flow inside the collection inlets from 8 to 12 have been given as follows.

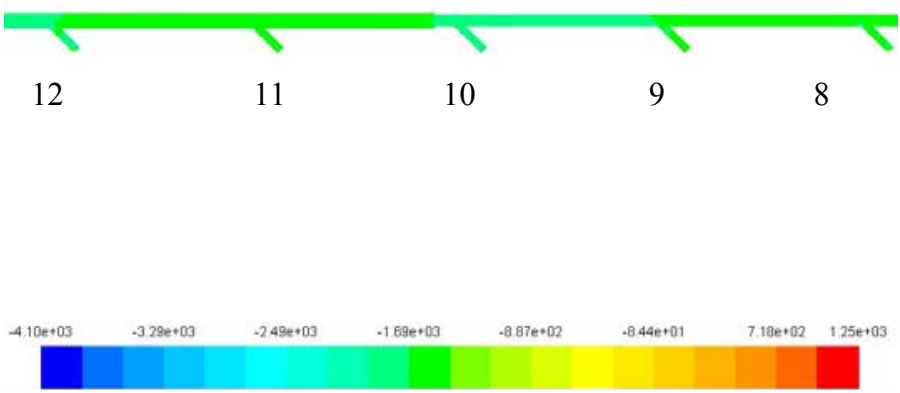

Fig. 10: Pressure contours for inlets 8-12 


\begin{tabular}{|c|c|c|c|c|c|c|}
\hline 12 & & 11 & 10 & & 9 & 8 \\
\hline $0.00 \mathrm{e} \cdot 00$ & $8.61 \mathrm{e}+00$ & $1.72 \mathrm{e}+01$ & $2.58 \mathrm{e} \cdot 01$ & $3.44 \mathrm{e}+01$ & $4.31 \mathrm{e} \cdot 01$ & $5.17 \mathrm{e}+01$ \\
\hline
\end{tabular}

Fig. 11: Velocity contours for inlets $8-12$

Figures 10 and 11 show the details of the static pressure and flow field of inlets from 1 to 12 on the condition that $1,4,8$ and 12 inlets were opened at a single time discharge. It was observed that the static pressure at first, fourth, eighth and twelfth inlets connection pipe was $1264.4,1288.68,1333.8$ and $1428.7 \mathrm{~Pa}$ respectively. It was clearly stated that the static pressure was higher to the nearest to the blower and gradually reduces the static pressure to the twelfth inlet point.

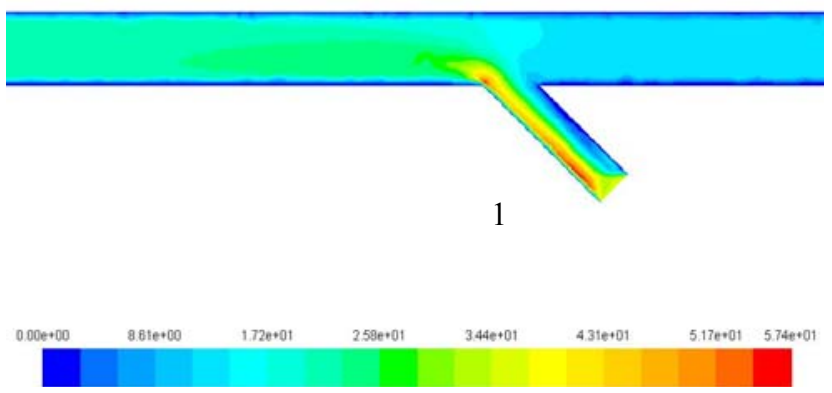

Fig. 12: Velocity contours for inlets $1-4-8-12$

As shown in Figures 12 and 13, the velocity at first, fourth, eighth and the twelfth inlet connection pipe was $12.3,20.89,29.84$ and $37.33 \mathrm{~m} / \mathrm{sec}$ respectively. It was also observed that the velocity gradually reduced from the " $y$ " junction to the main pipe. 


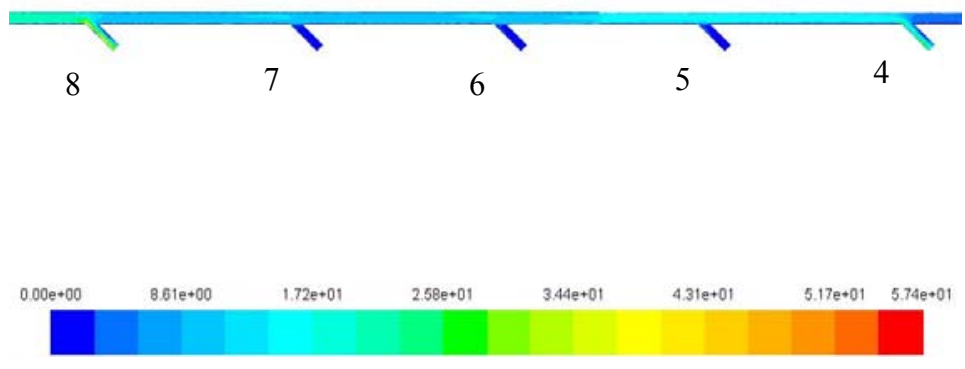

Fig. 13: Velocity contours for inlet point $4-8$

\section{Results and Discussions}

The simulation results of the current centralized collection system along with $5 \mathrm{Hp}$ Blower $(2070 \mathrm{~Pa})$ and the inlets 1 , inlets $1 \& 4$, inlets $1,4 \& 8$ and inlets $1,4,8 \& 12$ were opened and the respective results have been tabulated in Table 1 .

Table 1: Numerical results for case 2 to 4

\begin{tabular}{|c|c|c|c|}
\hline Case No & Open condition & $\begin{array}{c}\text { Velocity at mouth of } \\
\text { "y" }(\mathrm{m} / \mathrm{sec})\end{array}$ & Pressure (Pascal) \\
\hline 2 & Inlet 1 & 76.39 & 948.77 \\
\hline 3 & Inlet 1 & 28.19 & 924.34 \\
\hline & Inlet 4 & 55.20 & 1272.3 \\
\hline 4 & Inlet 1 & 15.22 & 1274.5 \\
\hline & Inlet 4 & 32.82 & 1340.6 \\
\hline & Inlet 8 & 44.27 & 1380.2 \\
\hline 5 & Inlet 1 & 12.37 & 1264.4 \\
\hline & Inlet 4 & 20.89 & 1288.6 \\
\hline & Inlet 8 & 29.84 & 1333.8 \\
\hline & Inlet 12 & 37.33 & 1428.7 \\
\hline
\end{tabular}

The Graph shows that the simulated velocity of four inlets $(1,4,8,12)$ were opened at a single time discharge. 


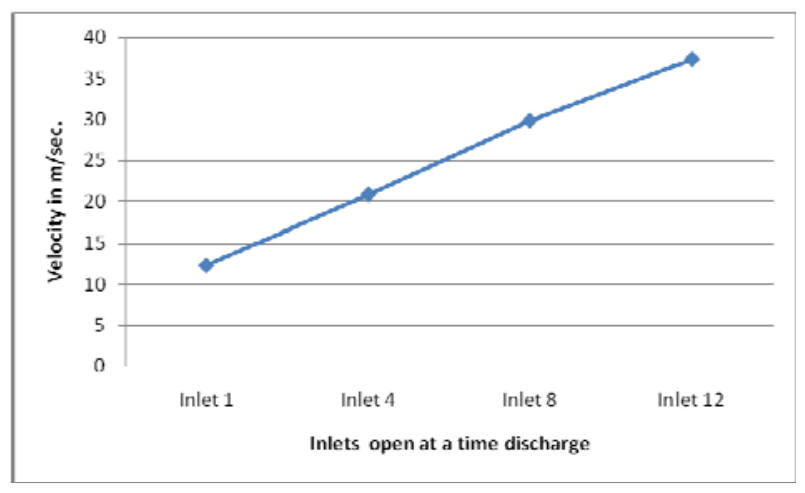

Fig. 14: Velocity of four inlet points are open

The simulation results of all inlet points from 1 to 12 were opened at a single time discharge have been tabulated in Table 2 .

Table 2: Numerical results for case 1

\begin{tabular}{|c|c|c|}
\hline Inlet points & Velocity at mouth of “y” $\mathrm{m} / \mathrm{sec}$ & Velocity at between "y" $\mathrm{m} / \mathrm{sec}$ \\
\hline 1 & 5.6 & 4.6 \\
\hline 2 & 8.9 & 7.8 \\
\hline 3 & 13.4 & 12.8 \\
\hline 4 & 14.9 & 15.7 \\
\hline 5 & 16.4 & 14.4 \\
\hline 6 & 26.8 & 25.2 \\
\hline 7 & 29.8 & 28.9 \\
\hline 8 & 32.9 & 29.5 \\
\hline 9 & 41.7 & 39.7 \\
\hline 10 & 44.6 & 42.8 \\
\hline 11 & 47.7 & 44.2 \\
\hline 12 & 55.2 & 54.3 \\
\hline
\end{tabular}

As per the previous study on current centralized collection system, the maximum power consumption results were observed through theoretical and experimental analysis for four inlets which were opened at a single time discharge. Hence, the maximum opening inlets were $1,4,8$ and 12 have been considered and the simulated results of middle velocity between the "y" joints have been tabulated.

Figure 15 shows that the simulated velocity of all inlets which were opened at a single time discharge. 


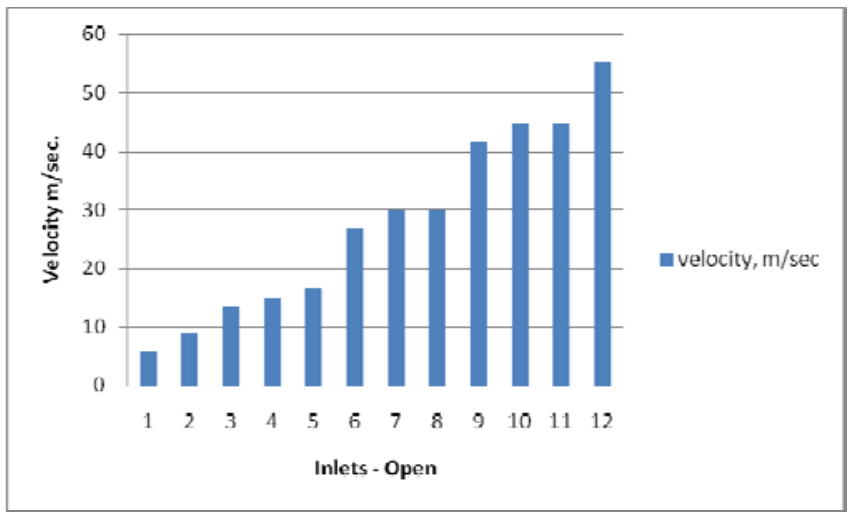

Figure 15: Velocity of all inlets

From the simulated results, it was clearly seen that the maximum four openings of inlets at a single time discharge of current $5 \mathrm{HP}$ blower was an effective collection of fluffs from the collection boxes. In order to confirm the optimum results of the centralized collection system, the experimental investigation of the above arrangements has to be carried out.

The numerical results of current 5 HP centralized collection system were summarized in Table 3.

Table 3: Numerical results of cases 2 to 5

\begin{tabular}{|l|l|l|}
\hline \multicolumn{1}{|c|}{$\begin{array}{c}\text { Open condition } \\
\text { Case 2 to 5 }\end{array}$} & \multicolumn{2}{c|}{ Nelocity $\mathrm{m} / \mathrm{s}$} \\
\hline Inlet 1 & 76.39 & 96.6 \\
\hline Inlet 1 & 20.19 & 94.2 \\
\hline 4 & 55.2 & 129.6 \\
\hline Inlet 1 & 15.72 & 129.9 \\
\hline 4 & 32.82 & 137.4 \\
\hline 8 & 44.27 & 140.6 \\
\hline Inlet 1 & 12.37 & 128.8 \\
\hline 4 & 20.89 & 131.3 \\
\hline 8 & 29.84 & 135.8 \\
\hline 12 & 37.33 & 145.5 \\
\hline
\end{tabular}

It was found that the velocity has been increased from inlets 1 to 8 in 1,4 and 8 inlets were opened at a time discharge. For inlets 1, 4, 8 and 12, the velocity has been 
increased from $12.37 \mathrm{~m} / \mathrm{sec}$ to 37.33 and corresponding pressure was increased from 131.3 to 145.5 mmwc.

A reasonable confirmation made with numerical investigation results on centralized collection system on the inlets $1,4,8$ and 12 was the maximum utilization of output power of the motor. If the four inlets exceeded to gather at a time discharge, the efficiency of the fluffs collection of the blower is reduced and same was observed in the previous experimental study. So, it was concluded that the four inlets which were opened at a single time discharge was the optimum condition for current centralized collection system (5Hp).

Accordingly, the experimental validation has to be carried out to compare the numerical results for optimization of centralized collection system for energy saving.

\section{Conclusions}

This numerical investigation contributes towards a better understanding of the principal fluid flow phenomena of a centralized waste collection system with various inlets opened at a single time discharge. The results of output parameters like velocity and pressure can be used for further design optimization of the centralized collection system to reduce the energy consumption.

In this study, the major parameters like the velocity and Total Pressure were analyzed and the following conclusions were made.

- The velocity and pressure in inlet 1 (case 2) was observed as $76.3 \mathrm{~m} / \mathrm{sec}$ and 948.8 $\mathrm{Pa}$ respectively at a time discharge.

- In inlets 1 and 4 (case 3), it was observed that the velocity and pressure was $28.19 \mathrm{~m} / \mathrm{sec}, 924.3 \mathrm{~Pa}$ and $55.2 \mathrm{~m} / \mathrm{sec}, 1272 \mathrm{~Pa}$ respectively.

- Similarly it was observed that the velocity in inlets $1,4,8$ and 12 ( case 5) was $12.37 \mathrm{~m} / \mathrm{s}, 20.89 \mathrm{~m} / \mathrm{s}, 29.84 \mathrm{~m} / \mathrm{s}$ and $37.3 \mathrm{~m} / \mathrm{s}$ respectively.

As per the previous theoretical analysis [16], it was observed that the maximum power consumption for four inlets $(1,4,8$ and 12) opened at a single time discharge was $3.45 \mathrm{kw}$ against the actual installed power of $3.7 \mathrm{kw}(5 \mathrm{Hp})$. Hence, the maximum opening inlets were $1,4,8$ and 12 have been considered.

It was concluded that the combination obtained using numerical results $(1,4,8$ and 12 opened at a time discharge) of velocity and pressure has been chosen as a input design parameters for the further analysis of centralised waste collection system to get an optimize alternative solution for better energy saving. The results of this study will surely help to analyse the centralized waste collection system for experimental validation.

\section{References}

[1]. S.M Yahya, Turbines Compressors and Fans. Tata Mc-Graw Hill Edition, 1988.

[2]. Atre, P.C., and T.R. Karuppa. Numerical Design and Parametric optimization of Centrifugal fans with Aerofoil blade Impellers. Research Journal of Recent Sciences, 2012;1(10): 7-11.

[3]. Mohammed Mohaideen, M. Optimization of Backward Curved Aerofoil Radial Fan Impeller using Finite element Modelling, Procedia Engineering, 2012; 38: 1592-1598.

[4]. Sheam Chyun Lin and Ming lun. Tsai, An Integrated Performance Analysis for a Backwardinclined Centrifugal Fan, Computers and Fluids, 2012; 56:24-38.

[5]. Srinivasa Rao,M. and N.A. Vallayil, Review of Simulation Approaches in Reliability and 
Availability Modeling, International Journal of Performability Engineering, 2016; 12(4): 369388.

[6]. Jazouli, P. Sandborn and A. Kashani pour. A Direct Method for Determining Design and Support Parameters to Meet an Availability Requirement, International Journal of Performability Engineering, 2014; 10(2): 211-225.

[7]. Krishna, V., K. Naresh Kumar and M. Prasanth Kumar, Numerical Analysis of Centrifugal Blower Using CFD, International Journal of Engineering Research \& Technology, 2013; 2(8): 1461-1463.

[8]. Walid Aniss Aissa1, Tarek Abdel Malak Mekhail, Soubhi Ali Hassanein and Osama Hamdy, Experimental and numerical Study of dilute Gas-Solid flow inside a $90^{\circ}$ horizontal square pipe bend, Open Journal of Fluid Dynamics, 2013; 3: 331-339.

[9]. Kaushik,V.V.R. S. Ghosh, G. Das, and Prasanta Kumar Das. CFD simulation of core annular flow through sudden contraction and expansion, Journal of Petroleum Science and Engineering, 2012; 86(87): 153-164.

[10].Jang, C.M, D.W. Kim and S.Y. Lee, Performance characteristics of turbo blower in a refuse collecting system according to operation conditions, Journal of Mechanical Science and Technology , 2008; 22: 1896-1901.

[11].Keyur k Patel and Prajesh M. Patel, Performance analysis and optimization of centrifugal fan, International Journal of Emerging Trends in Engineering and Development, 2013; 3(2): 261270.

[12]. Sushant Wagh., and Dhananjay Panchagade. Analyzing effect of impeller width on mass flow rate of Centrifugal Blower using CFD, International Journal of Engineering and Advanced Technology, 2014; 3(5): 236-238.

[13]. Ragoth Singh., and R M Nataraj, Optimizing impeller geometry for performance enhancement of a centrifugal blower using the Taguchi quality concept, International Journal of Engineering Science and Technology, 2012; 4(10): 4308-4314.

[14]. Mojtaba Gholamian., Gurram Krishna Mohan Rao., and Bhramara Panitapu. Effect of axial gap between inlet nozzle and impeller on efficiency and flow pattern in centrifugal fans, Case Studies in Thermal Engineering 1, 2013; 26-37.

[15].Jayapragasan. C.N., and K. Janardhan Reddy, Design Verification and Experiemtnal Aanalysis on Industrial Cleaner for Fluffs Collection, International Journal of Performability Engineering, 2016; 12(5): 459-470.

[16]. Jayapragasan. C.N, Experimental Analysis of centralized collection blower in spinning plant, Apm united Group, Practical Approach, 2015; 240-248.

Jayapragasan. C.N is an Assistant General Manager, Group R\&D at APM UG group of companies. He is a Research Scholar of VIT university, Chennai, Tamilnadu, India.

Dr. K. Janardhan Reddy is Professor and Dean of the School of Mechanical and Building Sciences at VIT University, Chennai, Tamilnadu, India. 